\title{
The Photon-Baryon Governed Universe
}

\author{
Laszlo A. Marosi \\ Paul-Klee-Str. 5, D-67061 Ludwigshafen am Rhein, Germany \\ Correspondence should be addressed to Laszlo A. Marosi, laszlomarosi@aol.com
}

Received 28 September 2011; Accepted 12 December 2011

Academic Editor: C. P. Singh

Copyright ( 2012 Laszlo A. Marosi. This is an open access article distributed under the Creative Commons Attribution License, which permits unrestricted use, distribution, and reproduction in any medium, provided the original work is properly cited.

\begin{abstract}
In a previous paper we postulated that the repulsive force responsible for the universal expansion is associated with the excitation of the empty space (quantum vacuum) and the excitation energy is represented by the energy of the cosmic microwave background $(\mathrm{CMB})$. In this paper, we show that the concept of the repulsive space expanding photon field (i) can successfully be applied to explain the local velocity anomaly of the Milky Way Galaxy as shown by Faber and Burstein (1998) and Tully (1998), (ii) offers a convincing explanation of the still disputed question of the cosmological expansion on local and intergalactic scales discussed by Cooperstock et al. (1998), and (iii) explains the redshift (RS) of the CMB in accordance with the law of energy conservation without the need for dark matter (DM) and dark energy (DE). Probably the most remarkable result of this model (abbreviated as photon/baryon: $\mathrm{PB}$ model in the following discussion) is that the individual voids building up the soup-bubble- (SB-) like galaxy distribution are the governing dynamical components of the universal expansion. Further consequence implies that the universe is considerably older than the interpretation of the Hubble constant as expansion velocity suggests.
\end{abstract}

\section{Introduction}

The cosmological constant $\Lambda$ was originally introduced by Einstein as a new term in his gravitational field equations in order to construct a static universe:

$$
R_{\mu \nu}-\frac{R g_{\mu \nu}}{2}+\Lambda g_{\mu \nu}=\frac{8 \pi G}{c^{4}} \times T_{\mu \nu}
$$

In (1) $R_{\mu \nu}-R g_{\mu \nu} / 2$ is the Einstein tensor and $T_{\mu \nu}$ is the energymomentum tensor and $\Lambda$ is the cosmological constant.

Einstein did not consider the cosmological constant to be part of the energy-momentum tensor [1]. The left hand side of (1) contains the metric tensor and its derivatives and the new constant $\Lambda$ appears in addition to this term. One can equally put $\Lambda$ on the right hand side of (1) and view the cosmological constant as an additional matter tensor

$$
T_{\mu \nu}^{*}=\frac{c^{4}}{8 \pi G} \times \Lambda g_{\mu \nu}
$$

and thus,

$$
R_{\mu \nu}-\frac{R g_{\mu \nu}}{2}=\frac{8 \pi G}{c^{4}}\left(T_{\mu \nu}+T_{\mu \nu}^{*}\right)
$$

More recently, it has become common [2] to interpret $\Lambda$ as a form of energy present in the empty space (vacuum energy) and consequently as part of the matter tensor $T_{\mu \nu}$.

Because general relativity (GR) never defined the exact nature of matter, in context with GR both interpretations could turn out to be more successful when applying GR to the universe as a whole [3]. In the following discussion we consider $\Lambda$ as an additional matter tensor $T_{\mu \nu}^{*}$ as defined in (2).

The physical nature of the cosmological constant is still a matter of speculations. In current astrophysics $\Lambda$ is usually interpreted as the energy contained in the empty space. Unfortunately, the estimated energy of the vacuum exceeds the value required by the lambda-cold dark matter $(\Lambda \mathrm{CDM})$ model by 120 orders of magnitude.

In order to overcome this problem in recent approaches several forms of varying cosmological constants were introduced in the Einstein equation [4-6] and a number of authors constructed models, in which specific decay laws are postulated for $\Lambda$. Examples for phenomenological $\Lambda$ decay laws are summarized in Overduin and Cooperstock [7]. These theories, however, are entirely speculative as much 
as it is not clear what the physical nature of the dark energy should be.

As a new approach to explain the physical nature of the variable cosmological constant we postulated [8] that the repulsive force responsible for the universal expansion is associated with the excitation of the quantum vacuum and the excitation energy is represented by the energy of the $\mathrm{CMB}$ (other sources of radiation, the energy of the diffuse background radiation (DBR), and the energy of neutrinos are neglected) and presented arguments that photons possibly could generate properties similar to those of a repulsive with $1 / R_{U}$ decaying scalar field. Light is regarded as a kind of excitation of the empty space that, as every energetically excited system, tends to relax to a state with lower energy, a less excited state, or the ground energy state by doing work against the gravitational attraction of the baryons.

Our hypothesis is not without precedent in modern astrophysics and, in a general sense, akin the decay of the false vacuum in inflationary cosmology. Without discussing details of this theory, in quantum field theory a false vacuum is an excited energy state of the true vacuum possessing a unique property [9]. The energy density of a false vacuum $\left(\rho_{E, V}\right)$ remains during expansion constant, and thus the energy of the system increases by $\Delta E=\rho_{E, V} \Delta V$. From this it follows that work must have been done in order to keep energy conservation; $\Delta E=\rho_{E, V} \Delta V=-p_{V} \Delta V$ ( $p_{V}$ is the pressure of the false vacuum). The system moves from a higher to a lower energy state by doing work in the expansion of the false vacuum bubble.

In both, in case of the false vacuum and in case of the postulated repulsive photon field as well, the source of force, exerting space-time expansion is the excitation energy of the empty space that relaxes to the ground energy state by doing work either in the expansion of the false vacuum bubble or, as in case of the space expanding photon field, against the gravitational attraction of the baryons.

The notion of space expanding photon field is new and, therefore, supporting reference can hardly be found in literature. In a foregoing paper Novello [10] expressed the idea that, under circumstances, the gravitational field created by the photons is repulsive. Recently, Labun and Rafelski [11] put forward the idea that the origin of DE is the excitation energy of the quantum vacuum. Brynjolfson [12], in a different context, proposed the existence of a repulsive force associated with photons.

Here we describe the expansion of the universe with respect to the repulsive, space expanding property of the photon field that plays the role of a time dependent with $1 / R$ decaying scalar field and assume that the attractive gravitational force of the baryons is exactly balanced by the repulsive force exerted by the photons. We analyze cosmological observations exclusively on the basis of observable quantities, that is, the average size $\left(R_{V}\right)$ of the voids, the observed mass and radiation densities $\rho_{M \text {,obs. }}$ and $\rho_{\lambda \text {,obs. }}$, and the experimentally confirmed Euclidean geometry of the universe, instead of introducing unknown particles and energy. We examine if this description agrees with cosmological observations.

\section{The Photon-Baryon-Governed Universe}

If we in (3) replace $\Lambda$ by $T_{\mu \nu}^{*}=\left(\left(c^{4} \times \kappa\right) / 8 \pi G\right) \times \Psi_{\mu \nu}$ the Friedmann solution for a flat universe obtains the following form:

$$
\left(\frac{\dot{R}}{R}\right)^{2}=\frac{2}{R^{2}} \frac{G M}{R}-\frac{2}{R^{2}} \frac{\kappa \psi}{R}=0,
$$

where $M=\rho_{M} \times V$ and $\Psi=\rho_{\lambda} \times V, \rho_{M}$ and $\rho_{\lambda}$ are the mass and radiation energy densities today, $\kappa$ is a constant converting the present radiation energy density into energy $\mathrm{g}^{-1}$. Its numerical value can be calculated to $\kappa=G \times \rho_{M} / \rho_{\lambda}=$ $10^{-25} \mathrm{~cm} \mathrm{~g}^{-1}$.

If one is interested in the energy of the photon term at any time $t$ of the expansion history, $\psi_{t}$ is to be calculated from $\Psi_{t}=\kappa \Psi / R_{t}$ and $\lambda_{t}=\lambda_{0} \times\left(R_{t} / R\right)$.

Equation (4) can equally be represented by

$$
\left(\frac{\dot{R}}{R}\right)^{2}=\frac{2}{R^{2}} \frac{G N_{B}}{N_{L} R}-\frac{2}{R^{2}} \frac{\kappa N_{\lambda} h c}{\lambda R_{0}}=0,
$$

where $N_{B}$ is the total number of baryons, $N_{\lambda}$ the total number of photons in the universe, $N_{L}$ is Loschmidt's number, and $R_{0}$ is the radius at time $t=0$.

The first term on the right hand side is equivalent to Einstein's matter tensor, containing matter and all forms of energy $/ c^{2}$ which are associated with a certain physical state of matter. The second term is a new energy term, containing those forms of energy which are considered to be a property of the excited empty space, the energy of photons, and neutrinos.

Equation (5) means that the energy balance in the universe is determined by the ratio of the baryons to the photons. According to the grand unifying theories the ratio $\Omega=N_{B} / N_{\lambda}$ is fixed by microphysics [13-15] and has nothing to do with initial conditions; the physical processes leading to the formation of baryons and photons with a fixed ratio $\Omega$ were governed by the law of energy conservation which requires that the total energy in the universe as measured by the space curvature, $(G M / R)-(\kappa \psi / R)$ must have zero net value and thus, the critical fine-tuning problem does not arise.

2.1. Expansion of the $P B$ Universe. In (4) the first time derivative of $R$ seems to vanish for all times implying a static universe without expansion.

The major problem with any such stationary state equilibrium is how to start the present expansion process. The PB model provides a plausible mechanism for the expansion started off in an apparently steady-state origin: Primordial underdensities generated during the early stage of evolution represent centers of expansion and are the seeds for formation of the cosmic large-scale structure. Because mass underdensities are regions of suppressed gravitational attraction in underdense regions the repulsive force exerted by the still homogeneous photon field overwhelms the gravitational attraction and such regions accordingly expand. 
Matter streams away from underdense expansion centers and collects on the surface and in interstices of a close packing of spheres.

The observed large-scale structure of the universe is in support of this expansion mechanism. Measuring the clustering of bright galaxies has shown that the 3-dimensional distribution of luminous matter has a SB-like appearance with the visible galaxies on the surface of the soap bubbles $[16,17]$. The galaxies are situated in walls, filaments, and dense nodes, forming a network which surrounds huge voids. The voids occur on scales of $100 \mathrm{Mpc}$ and are free of matter. The excess of radiation energy inside empty spaces causes a true instability which can drive the cosmic expansion due to the postulated repulsive force of radiation.

In the following discussion we regard a single void as a representative part of the infinite universe and describe the universal expansion by the example of this isolated but representative sample. It is assumed that all voids of the universe expand in the same way.

The numerical value of $(G M / R)-(\kappa \Psi / R)$ in (4) can easily be calculated using Newtonian mechanics. We consider first a uniform homogeneous and isotropic solid sphere from the size of a void with radius $R_{S}$ and mass $M_{S}$. The gravitational self-energy of the solid sphere, US ( $\mathrm{ergs} \mathrm{g}^{-1}$ ) amounts to $0.6 \times G M_{S} / R_{S}$. We assume that this energy is exactly balanced by the energy of the repulsive photon field.

The gravitational self-energy of the corresponding thin spherical shell (void) with mass $M_{V}=M_{S}$ and radius $R_{V}=$ $R_{S}$ is $0.5 \times G M_{V} / R_{V}$. Because the homogeneous photon distribution has not changed, the energy of the repulsive photon field inside the void overwhelms the gravitational attraction by $0.1 \times G M_{V} / R_{V}$ and the bubble accordingly expands.

The expansion velocity is given by

$$
v_{\text {Void }}^{2}=\frac{2(0.6 \times G M-S \times 0.5 \times G M)}{R_{V}},
$$

where $v_{\text {Void }}$ is the expansion velocity of the void.

The structure or morphological factors $S$ takes into account that the gravitational potential energy ergs $\mathrm{g}^{-1}$ in the interior of a thin spherical shell with wall thickness $d$ and mass $M_{v}$ depends on $d$ :

$$
S=\int_{x=r_{v}-d}^{x=r_{v}} \frac{x^{3}-(x-1)^{3}}{x} \rho_{\text {shell }} d x,
$$

where

$$
\rho_{\text {shell }}=\frac{r_{v}^{3}}{r_{v}^{3}-\left(r_{v}-d\right)^{3}}\left(m_{v}=1, \frac{4 \pi}{3}=1\right) .
$$

From (7) and (8) the structure factor can be calculated to $S=$ 1.0254, 1.0516, 1.0787 for shells with thickness of $d=0.05$, $0.10,0.15 r_{v}$, respectively.

\section{Voids Really Push: The Local Velocity Anomaly}

The Local Void was identified in the Nearby Galaxies Atlas [18]. The empty region begins at the edge of the Local Group and our Milky Way lies on the surface of the Local Void. The size of the void is poorly defined, because much of it lies behind the plane of the Milky Way. It nearest part may have a diameter of $\sim 45-65 \mathrm{Mpc}[19,20]$ and the south and north extensions have diameter of 140 and $160 \mathrm{Mpc}$, respectively.

The local velocity anomaly was first noticed by Faber and Burstein [21] and Tully [22] and has subsequently been discussed by several authors [23-25]. Recently, a comprehensive study on this topic was published by Tully [26].

The observation is the following [26]. The Sun orbits in our Milky Way Galaxy at $220 \mathrm{~km} / \mathrm{s}$. The Milky Way is falling toward Andromeda Galaxy at $135 \mathrm{~km} / \mathrm{s}$. The neighborhood of our Galaxy is retarded from the cosmic expansion by the Virgo Cluster by $185 \mathrm{~km} / \mathrm{s}$. These velocities correspond to the expected gravitational attraction by the mass of the neighboring galaxies.

However, after subtraction of the velocity components caused by the nearby galaxies, the residual pattern of motion reveals an extra component. The Milky Way moves away from the Local Void with a velocity of $260 \mathrm{~km} / \mathrm{s}$ in direction close to the supergalactic South Pole. This movement is not toward anything substantial, but it is directly away from the Local Void, orthogonal to the disk of the Local Sheet [26].

It was suggested that this offset might be the consequence of a push from the Local Void [27], whereas concentrations of matter pull, a void pushes.

\section{Expansion of Voids according to the PB Model}

4.1. Comparison with the Local Velocity Anomaly. Using (6) we can now compare the observed local velocity of the Milky Way with the rate of expansion of the individual voids as inferred from the interaction of the postulated photonbaryon-induced repulsive and attractive forces, respectively.

The linear relationship between expansion velocities with increasing radii of the voids is shown in Figure 1.

Results for voids with radii of $33,50,66$, and $80 \mathrm{Mpc}$, $S=1.0254$, and $\rho_{M}=6 \times 10^{-31} \mathrm{~g} \mathrm{~cm}^{-3}$ are summarized in Table 1.

The expansion velocities in Table 1 lead to a Hubble constant of $5.54 \mathrm{~km} \mathrm{~s}^{-1} \mathrm{Mpc}^{-1}$.

It can be seen from Table 1 that voids really generate a push in range of the observed local velocity.

This result could have far-reaching consequences for our understanding of the universal expansion. Regarding voids as the dynamical component of the expansion, the largescale structure can be thought of as a close packing of expanding spheres of different sizes and the linearity of the individual expansion rates with distance makes it impossible to differentiate local void expansion from a global cosmic expansion.

4.2. Age of the Universe. The PB model implies a spectacular outcome for the age of the universe. The time necessary for 


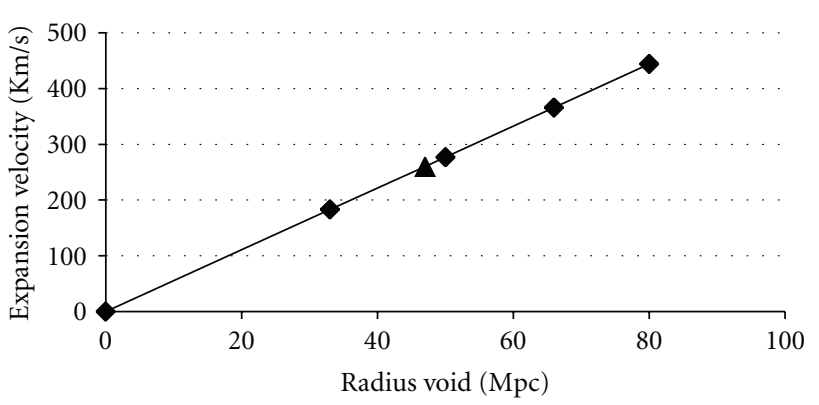

FIgURE 1: Expansion velocity of voids with increasing radii. The triangle represents the local velocity of the Milky Way Galaxy orthogonal to the disk of the local sheet.

TABLe 1: Expansion velocity $V\left(\mathrm{~km} \mathrm{~s}^{-1}\right)$ of voids with radii 33, 50, 66 , and $80 \mathrm{Mpc}$.

\begin{tabular}{lcccc}
\hline$R_{v}(\mathrm{Mpc})$ & 33 & 50 & 66 & 80 \\
$M_{\text {Shell }}(\mathrm{g})$ & $2.512 \times 10^{48}$ & $8.737 \times 10^{48}$ & $2.008 \times 10^{49}$ & $3.56 \times 10^{49}$ \\
$V\left(\mathrm{~km} \mathrm{~s}^{-1}\right)$ & 183 & 277 & 366 & 444 \\
\hline
\end{tabular}

the expansion of a primal void with $R \ll 10^{26} \mathrm{~cm}$ to an average size void of $10^{26} \mathrm{~cm}$ can be obtained by integration of $d t=\sqrt{R /\left(2(0.6-S \times 0.5) G \times M_{V}\right)} d R$ :

$$
t=\frac{2}{3 \sqrt{2(0.6-S \times 0.5) \times G M_{V}}}\left(10^{26}\right)^{3 / 2} .
$$

From (9) with $\rho_{M}=6 \times 10^{-31} \mathrm{~g} \mathrm{~cm}^{-3}$ the time necessary for expansion to a radius of $10^{26} \mathrm{~cm}$ can be inferred to about 115 Billion years. The $\mathrm{PB}$ model, predicting an age of the universe of $\gg 115$ billion years, could help to solve the age problem of galaxy formation by using the idea that the universe is much older, and so they may have been evolved over time in some regular way without the need for DM or DE.

\section{Cosmological Expansions on Local and Intergalactic Scales}

The question as to whether the universal expansion affects local systems is still a matter of debate and during the last decades several papers appeared in scientific literature dealing with this issue. On basis of a detailed quantitative analysis, Cooperstock et al. [28] came to the conclusion that the effect of expansion due to the force of the Hubble flow does not affect local systems and is negligible even for clusters of galaxies. Galaxies and clusters of galaxies do not partake of expansion because the internal gravity of a galaxy or galaxy cluster is stronger than the force of the expansion due to the Hubble flow. Cooperstock et al. concluded that expansion of space occurs strictly between galaxies and galaxy clusters.

In a more recent paper, Gentry [29] pointed out that the gravitational force between two spherical galaxy clusters with $M=10^{47} \mathrm{~g}$ and distance of $\sim 10^{26} \mathrm{~cm}$ is $10^{10}$ times stronger than that between the solar system and the Milky Way galaxy. With this objection the question of global expansion remains obscure and a definitive answer to this issue is still lacking.
The PB model offers a more convincing explanation of the expansion on intergalactic and on local scales. We have shown that according to the PB model the universe can be thought of as consisting of underdense, expanding, and overdense, contracting, regions. An important feature of the SB universe is that due to its still homogeneous and isotropic large-scale structure, the amount of mass $m_{\text {shell }}$ contained in the thin outer shell of the voids corresponds to $m_{\text {shell }}=V_{\text {Void }} \times \rho_{M}\left(\rho_{M}=\right.$ mean mass density in the universe). It has been shown that inside of empty spaces the force of the repulsive photon field overwhelms the gravitational attraction if $m_{\text {shell }} \leq V_{\text {Void }} \times \rho_{M}$ and the empty spaces accordingly expand. In galactic systems with $M_{G} \gg$ $V_{G} \times \rho_{M}$ the attractive gravitational potential predominates and consequently, galactic systems are not subject to the universal expansion. In our model remote galactic systems are separated by huge expanding empty spaces and there is no effective gravitational attraction between distant galaxies. Therefore, the objection given in [29] is not valid.

\section{Redshift of the CMB}

With the postulate of the PB model the energy decrease of the $\mathrm{CMB}$ is equal to the work done by the photons in the expansion of the universe versus the gravitational attraction of the baryons:

$$
\Delta E_{\lambda}=-\int_{R_{1}}^{R_{2}} \frac{G M^{2}}{R^{2}} d R=-\left(\frac{G M^{2}}{R_{1}}-\frac{G M^{2}}{R_{2}}\right)
$$

and with (10)

$$
Z=\frac{\lambda_{2}-\lambda_{1}}{\lambda_{1}}=\frac{E_{\lambda}^{r 1}}{E_{\lambda}^{r 2}}-1=\frac{G M^{2} / R_{1}}{G M^{2} / R_{2}}-1=\frac{R_{2}}{R_{1}}-1 .
$$

Equation (11) shows that increase in the wavelengths of the photons is proportional to the change of the scale factor.

However, the value of the Hubble constant of $5.54 \mathrm{~km} \mathrm{~s}^{-1} \mathrm{Mpc}^{-1}$ is far not enough to explain the RS of atomic spectral lines. A resolution of this problem might be found in the hypothesis that the RS of spectral lines is composed of a velocity and a superimposed RS component of as yet unknown origin and the rate of expansion is much lower, say, the universe expands according to the prediction of the PB model. A new RS mechanism based on the process of energy equilibration of starlight into the equilibrium blackbody radiation for explaining the Hubble relation is developed in order to explain the RS of atomic spectral lines [30]. An important feature of this theory is that the energy of the redshifted spectral photons is merely converted into CMB energy and there is no real loss of energy associated with this part of the RS because the total energy $\left(E_{\text {starlight }}+E_{\mathrm{CMB}}\right)$ has not changed.

\section{Conclusions}

The results presented in this paper have demonstrated that the PB model, describing the evolution of the universe from the interaction of attractive and repulsive forces, can 
explain (i) the fine-tuning or flatness problem, (ii) the age problem in galaxy formation without the need for DM and $\mathrm{DE}$, and (iii) the loss of energy of the CMB in accordance with the law of energy conservation. One advantage that the PB model would have over $\Lambda$ CDM is that it works with constituents that are known to be present in the universe today. Probably the most remarkable cosmological implication of the results presented in this paper is that the individual voids building up the SB-like galaxy distribution are the governing dynamical component of the universal expansion. Voids expand and the overdense regions collapse.

An observational test of this model could be made by determining the radial velocities of galaxies situated on the border of voids and comparing these velocities with those obtained from our model.

\section{Acknowledgment}

The author is grateful to Professor Rainer Mattes from the Westfälische Wilhelms-Universität, Münster, Germany, for proofreading and for his continuous interest in this work.

\section{References}

[1] P. J. E. Peebles and B. Ratra, "The cosmological constant and dark energy," Reviews of Modern Physics, vol. 75, no. 2, pp. 559-606, 2003.

[2] C. W. Misner, K. S. Thorne, and J. A. Wheeler, Gravitation, W. H. Freeman, New York, NY, USA, 1973.

[3] E. R. Harrison, Cosmology, The Science of the Universe, Cambridge University Press, 1981.

[4] R. R. Caldwell, R. Dave, and P. J. Steinhardt, "Cosmological imprint of an energy component with general equation of state," Physical Review Letters, vol. 80, no. 8, pp. 1582-1585, 1998.

[5] P. J. E. Peebles and B. Ratra, "Cosmology with a Time-Variable Cosmological "Constant", Astrophys Journal Letters, vol. 325, p. L17, 1998.

[6] P. J. Steinhardt, L. Wang, and I. Zlatev, "Cosmological tracking solutions," Physical Review D, vol. 59, no. 12, Article ID 123504, 13 pages, 1999.

[7] J. M. Overduin and F. I. Cooperstock, "Evolution of the scale factor with a variable cosmological term," Physical Review D, vol. 58, no. 4, Article ID 043506, 23 pages, 1998.

[8] L. A. Marosi, "Influence of the Soup-Bubble Structure on the Stability of a Static, Flat Universe Consisting of Matter and a Repulsive with 1/R Decaying Scalar Field," Apeiron, vol. 15, no. 2, p. 528, 2008.

[9] P. C. B. Davies, The New Physics, Cambridge University Press, 1989.

[10] M. Novello, “Cosmic repulsion,” Physics Letters A, vol. 90, no. 7, pp. 347-348, 1982.

[11] L. Labun and J. Rafelski, "Vacuum structure and dark energy," International Journal of Modern Physics D, vol. 19, no. 14, pp. 2299-2304, 2010.

[12] A. Brynjolfson, "Plasma Redshift," in the American Physical Society Meeting, May 2009.

[13] M. Yoshimura, "Unified gauge theories and the baryon number of the universe," Physical Review Letters, vol. 41, no. 5, pp. 281-284, 1978.
[14] S. Weinberg, "Cosmological production of baryons," Physical Review Letters, vol. 42, no. 13, pp. 850-853, 1979.

[15] A. Zee, "Horizon problem and the broken-symmetric theory of gravity," Physical Review Letters, vol. 44, no. 11, pp. 703-706, 1980.

[16] P. J. E. Peebles, The Large Scale Structure of the Universe, Princeton University Press, 1980.

[17] S. D. Landy, "Mapping the universe," Scientific American, pp. $38-45,1999$.

[18] R. B. Tully and J. R. Fisher, Nearby Galaxy Atlas, Cambridge University Press, 1987.

[19] R. B. Tully, arXiv:0708.2449v1, 2007.

[20] "Major Voids in the Universe," http://www.atlasoftheuniverse.com/nearsc.html.

[21] S. M. Faber and D. Burstein, "Motion of galaxies in the neighborhood of the local group," in Large Scale Motions in the Universe: Vatican Study Week, V. C. Rubin and G. V. Coyne, Eds., p. 116, Princeton University Press, Princeton, NJ, USA, 1998.

[22] R. B. Tully, "Motion of Galaxies in the Neighborhood of the Local Group," in Large Scale Motions in the Universe: Vatican Study Week, V. C. Rubin and G. V. Coyne, Eds., p. 169, Princeton University Press, Princeton, NJ, USA, 1998.

[23] E. Giraud, "The local anomaly of the extragalactic velocity field," A\&A, vol. 231, p. 1, 1990.

[24] M. Han and J. Mould, "The velocity field in the local supercluster," Astrophysical Journal, vol. 360, no. 2, pp. 448464, 1990.

[25] R. B. Tully, E. J. Shaya, and M. J. Pierce, "Nearby galaxy flows modeled by the light distribution: distances, model, and the local velocity anomaly," Astrophysical Journal, Supplement Series, vol. 80, no. 2, pp. 479-499, 1992.

[26] R. B. Tully, “The Local Void is Really Empty,” arXiv:0708.0864, 2007.

[27] R. C. Kraan-Korteweg and O. Lahav, "The universe behind the Milky Way," Astronomy and Astrophysics Review, vol. 10, no. 3, pp. 211-261, 2000.

[28] F. I. Cooperstock, V. Faraoni, and D. N. Vollick, "The influence of the cosmological expansion on local systems," Astrophysical Journal, vol. 503, no. 1, pp. 61-66, 1998.

[29] R. V. Gentry, "Galaxies Point to Flaws in Big Bang's Expanding-Balloon Illustration and to Smoking Gun Signatures of GENESIS," arXive: physics/0102094, 2001.

[30] L. A. Marosi, "Does The Hubble Constant Really Represent Recession Velocity?” submitted for publication. 

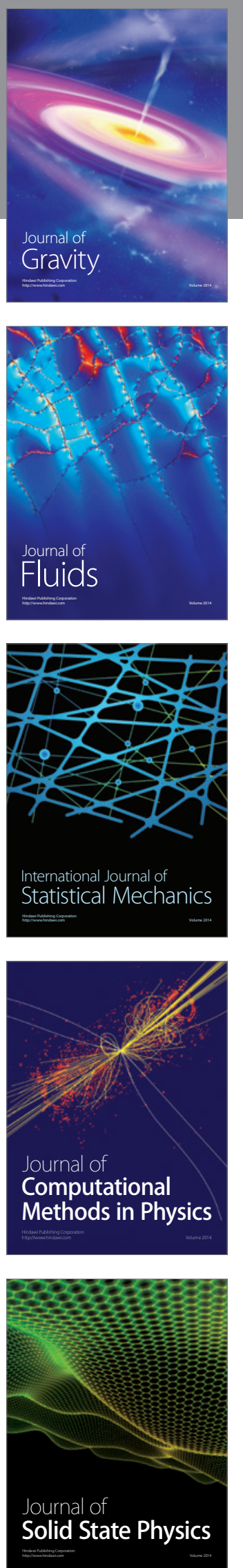

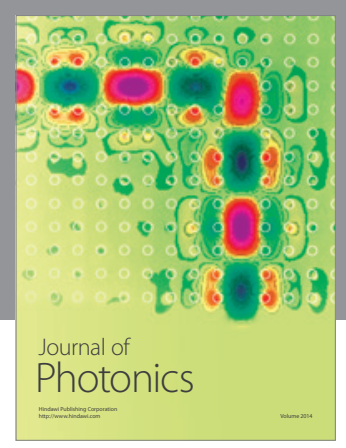

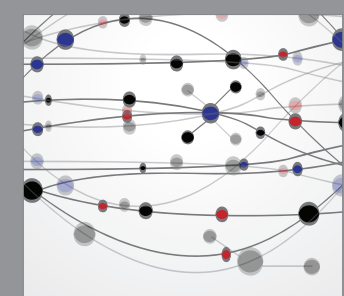

The Scientific World Journal
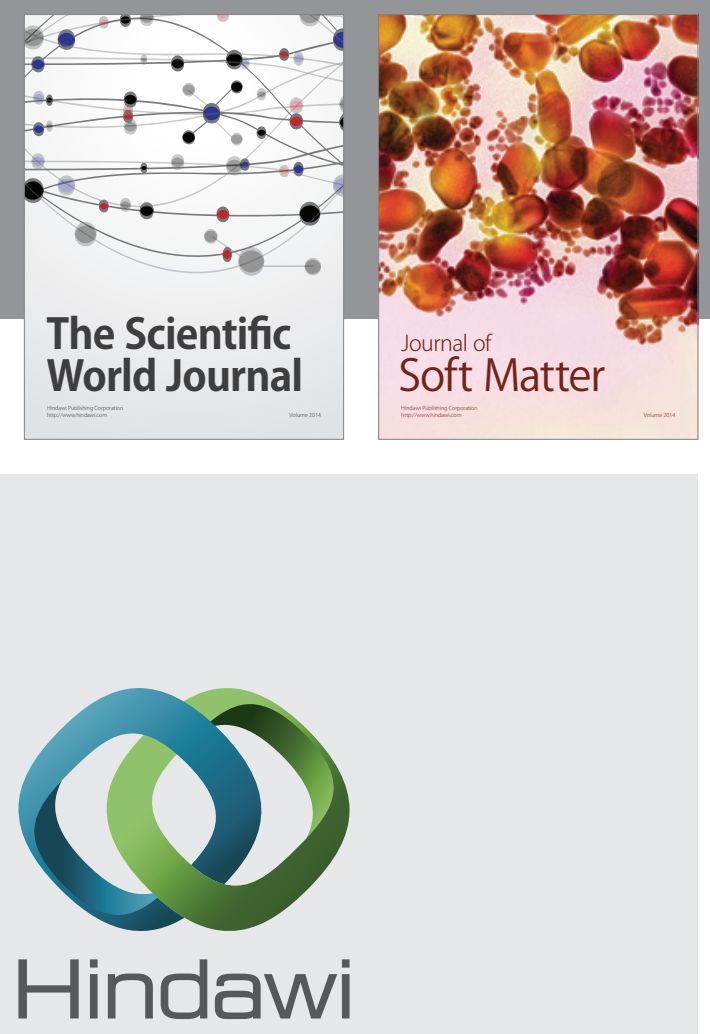

Submit your manuscripts at

http://www.hindawi.com
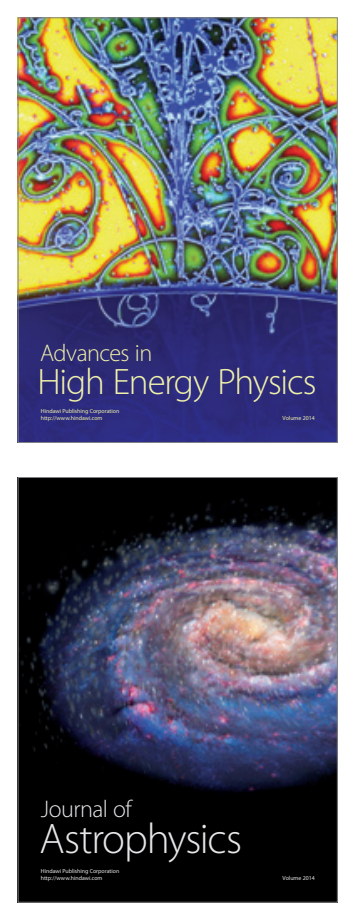
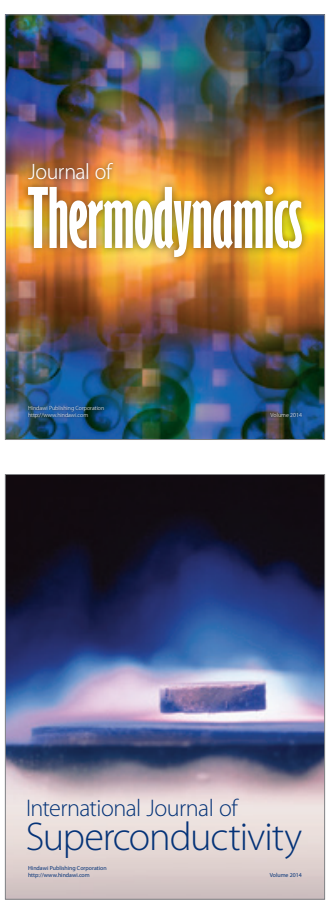
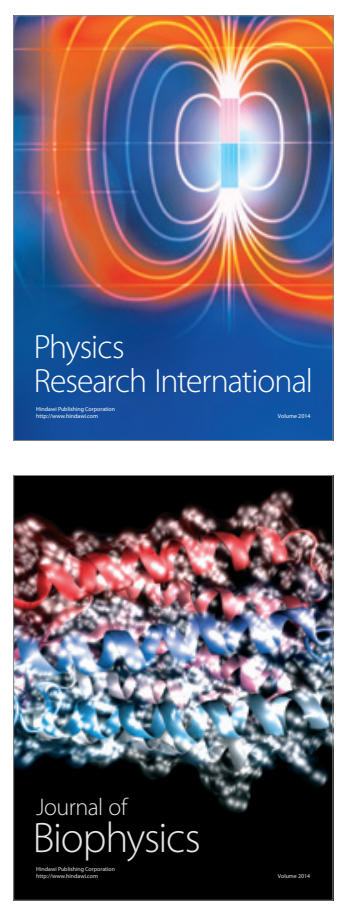
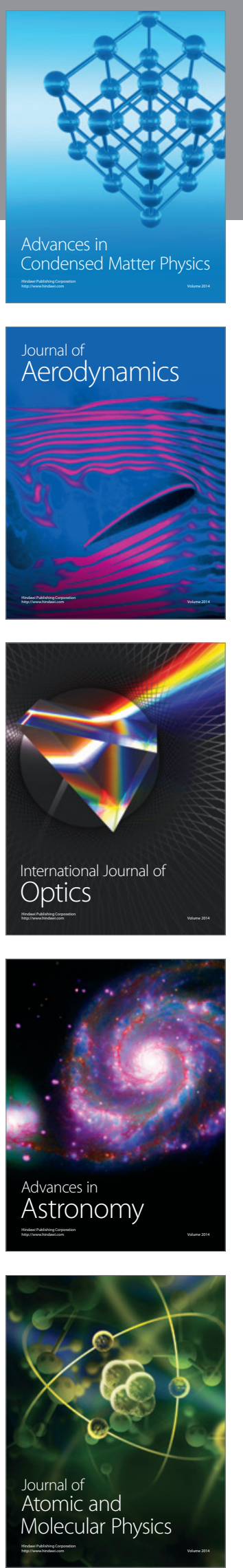